\title{
Biologie moléculaire de l'éruption dentaire : mise au point
}

\author{
The molecular biology of tooth eruption: what have we learnt?
}

\author{
ISABELLE NORMAND DE LA TRANCHADE, J AVOTTE NANCY
}

\begin{abstract}
RÉSUMÉ
Les étapes physiologiques de l'éruption dentaire sont parfaitement décrites et connues. Cependant les mécanismes moléculaires et biologiques qui concourent à faire évoluer la dent hors de son site intra-osseux restent encore mal compris. L'éruption dentaire requiert une résorption osseuse localisée qui détermine le chemin d'éruption. Elle nécessite aussi une ostéogenèse comblant l'espace précédemment occupé par le germe dentaire. Contrairement aux anciennes hypothèses, la dent ne participe pas activement à sa propre éruption. En effet, si l'on substitue à la dent avant le début du processus d'éruption, une réplique réalisée en matériau inerte, celle-ci fait son éruption. L'intégrité du follicule dentaire est par contre indispensable. En effet, très tôt, le follicule dentaire va attirer dans sa partie coronaire des monocytes précurseurs des ostéoclastes. La partie apicale du follicule est le siège d'une prolifération cellulaire importante. La résorption-apposition osseuse est contrôlée par une série de facteurs d'éruption qui agissent d'une part en améliorant le recrutement monocytaire et en facilitant leur différenciation en ostéoclastes dans la région coronaire, d'autre part en stimulant les ostéoblastes dans la région apicale. Le follicule dentaire va produire et réguler les facteurs nécessaires à l'éruption. Ainsi, le facteur de croissance Epidermal Growth Factor (EGF) est impliqué dans la résorption osseuse, notamment en tant qu'activateur de la production du Transforming Growth Factor ß (TGF-ß). Ce dernier constitue un agent chimiotactique pour les monocytes et améliore l'expression de I'Interleukine-1 $\alpha$ qui stimule l'expression du Colony Stimulating Factor-1 (CSF-1), la molécule clef du chimiotactisme monocytaire. Le CSF-1, en injection intramaxillaire chez le rat ostéopétrotique, restaure l'éruption dentaire. Il stimule la production de Monocyte Chemotactic Protein-1 (MCP-1) et C-fos. MCP-1 joue un rôle semblable à celui de CSF-1. La Parathyroïde Hormone related Protein améliore l'expression de CSF-1 et MCP-1. Elle est nécessaire à la formation et à la différentiation des ostéoclastes. Enfin, c-fos est indispensable à la différenciation des ostéoclastes. Une meilleure connaissance de la biologie moléculaire locale au moment de l'éruption devrait permettre de trouver des solutions innovantes pour le traitement des dents incluses ou ayant un retard d'évolution ainsi que dans la maîtrise du turn-over osseux en parodontologie comme en orthodontie.
\end{abstract} (Med Buccale Chir Buccale 2003; 9: 95-103)

mots clés : Eruption dentaire, Formation osseuse, Résorption osseuse, Facteurs de croissance

\section{SUMMARY}

The physiological stages of dental eruption are well known and documented. However, the biological and molecular mechanisms that work together in the tooth's evolution outside the bone cavity, are still not well understood. Dental eruption requires a localized bone resorption in order to obtain an eruption pathway. It also requires an osteogenesis to fill in the cavity that the dental germ initially occupied. Contrary to conventional wisdom, the tooth itself does not parcipate actively in its own eruption. In fact, if the tooth is replaced by an inert object prior to eruption, this object still erupts. The integrity of the dental follicle is obligatory. In fact, early on, their is an influx of mononuclear cells, the osteoclast precursors, in the coronal region of the follicle. At the same time, a high level of cellular proliferation takes place in the apical part of the follicle The bone apposition and resorption is

* Faculté d'Odontologie - Université de Bordeaux II - 33000 Bordeaux - France

Demande de tirés à part:

* Normand de la Tranchade Isabelle 7 rue Roquelaine 31000 Toulouse

Article reçu le 4 juillet 2002. Accepté pour publication le 15 janvier 2003 
controled by a series of eruption factors that improve the monocyte influx and facilitate their differentiation in osteoclasts in the coronal part of the dental follicle on the one hand, and by stimulating the osteoblasts at the apical part on the other hand. Therefore, the dental follicule produces and regulates the factors needed for eruption. The Epidermal Growth Factor is thus implicated in bone resorption, especially to improve the production of Transforming Growth Factor - $\beta$. The latter is chemotactic for monocytes and improves the expression of Interleukine-1 $\alpha$. Monocyte Chemotactic Protein-1 plays a role similar to CSF-1. ParaThyroïde Hormone related Protein improves the expression of CSF-1 and MCP-1. PTHrP is necessary for the development and the differentiation of osteoclasts. Finally, c-fos is essential in the differentiation of osteoclasts. Osteopetrotic c-fos mutant mice have a lack of c-fos gene and have unerupted teeth. Eruption occures if c-fos is provided by an ectopic source. Understanding the local molecular biology of eruption should in the near future, lead to innovative applications in the treatment of unerupted teeth or delayed eruption as well as controling bone turnover in both parodontology and orthodontics. (Med Buccale Chir Buccale 2003; 9: 95-103)

key words : Tooth eruption, Bone formation, Bone eruption, Growth factors

De nombreux processus du développement n'ont toujours pas été totalement élucidés du point de vue moléculaire. L'éruption dentaire en fait partie. Comment un ensemble de tissus d'origine embryologique et à vocation différentes, mais à finalité unique, parvient-il à se dégager de sa position intra-osseuse ? Le mouvement de la dent pendant l'éruption nécessite la création d'un passage à travers l'os et les tissus mous. Pour l'éruption des dents définitives, la situation est encore plus complexe car on assiste en même temps à la rhizalyse des dents temporaires. La formation de ce chemin d'éruption constitue l'étape principale pendant laquelle vont intervenir de nombreux facteurs cellulaires et moléculaires. L'édification radiculaire est facilitée durant l'éruption, mais elle n'est plus considérée comme la cause majeure de ce processus. Quant au desmodonte, il contribue par son turn-over important à la stabilité de la dent fonctionnelle mais il ne semble guère intervenir dans l'éruption. Enfin, la formation d'os et de cément dans la région apicale maintient un mouvement éruptif lent permettant de compenser l'attrition dentaire.

\section{LES PHENOMENES CELLULAIRES DE L'ERUPTION DENTAIRE}

En 1969, Cahill cherche à empêcher l'éruption des prémolaires mandibulaires de chien en les transvissant [1]. II observe que cela n'empêche pas la formation d'un chemin d'éruption et que, lorsqu'il dépose les vis, l'éruption s'effectue. Cette expérience relance la controverse et pose de nouvelles questions :

Le processus d'éruption nécessite-t-il la présence de la dent ? Comment la formation du chemin d'éruption et les mouvements dentaires sont-ils régulés? Quels sont les tissus nécessaires à l'éruption?

\section{Rôle du follicule dentaire}

Cahill et Marks, en 1980 et 1982, réalisent les expériences suivantes [2,3]. Ils résèquent différentes structures d'une prémolaire de chien juste avant le début de son éruption, pour étudier les conséquences radiologiques et histologiques de ces ablations. L'ablation chirurgicale du gubernaculum dentis, ou de la racine en formation, ou encore de la couronne ne gênent pas la formation du chemin d'éruption ni la formation osseuse à la base de la crypte contenant le germe. Seule l'ablation du follicule inhibe l'éruption. Ces expériences mettent en évidence l'importance du follicule dentaire qui semble influencer, voire coordonner le processus d'éruption. Les expériences d'ablation du follicule sont reprises par Larson et coll. qui obtiennent les mêmes résultats [4]. II reste à déterminer les mécanismes cellulaires ayant lieu dans le follicule lors de l'éruption et les molécules qui les régulent. 


\section{Rôle de la résorption osseuse}

Parallèlement aux travaux précédents, Marks cherche à montrer que l'éruption dentaire est en étroite relation avec un processus d'ostéolyse [5]. Marks, lizuka et Cielinski ont été les principaux chercheurs à mener des expériences sur les rats ostéopétrotiques qui n'ont pas une éruption dentaire normale $[5,6,7]$. Ces rats ont des ostéoclastes incompétents, incapables de réaliser la lyse osseuse, ce qui semble empêcher l'éruption de leurs dents. L'expérimentation sur les rats ostéopétrotiques représente une précieuse source d'informations pour comprendre le phénomène d'ostéoclasie. L'ostéopétrose est une affection héréditaire, retrouvée chez de nombreux mammifères, y compris I'homme. Le trouble de la résorption osseuse entraîne des anomalies osseuses avec comblement des cavités médullaires, associées à un retard, voire une absence d'éruption. L'ostéopétrose serait due à une atteinte soit de la lignée des ostéoclastes, soit des cellules mésenchymateuses constituant le micro-environnement supportant le développement et l'activité des ostéoclastes. Dans le second cas, le traitement est complexe car il peut s'agir d'une altération d'un des nombreux facteurs intervenant dans l'éruption [8]. II existe plusieurs formes d'ostéopétrose et les troubles de l'éruption vont d'un simple retard à l'absence totale. Le caractère polymorphe de cette affection héréditaire suggère l'existence de plusieurs variantes génétiques.

Quant à Marks, il utilise pour son étude des rats ostéopétrotiques de la variété «incisor absent » [i a] qui présente une absence d'éruption des incisives et une éruption très retardée, voire une absence d'éruption, des molaires [5]. II irradie des rats nouveau-nés et observe l'éruption de leurs dents ainsi que la lyse osseuse, après trente jours. Pour les rats [i a] irradiés, le taux d'éruption des molaires est plus important que pour les rats $[i$ a] non traités, mais il reste inférieur à celui des rats normaux. II observe aussi que 48 heures après l'irradiation, il existe deux types d'ostéoclastes: des ostéoclastes comparables à ceux trouvés chez les rats [i a] non traités et des ostéoclastes normaux. II en conclut que les troubles de l'éruption pour les rats [i a] seraient directement liés à la diminution de la lyse osseuse.

En 1983, Marks et coll. notent la présence de cellules mononuclées au début de l'éruption en position juxta-vasculaire, dans la région coronaire du follicule dentaire [9]. Leur nombre augmente juste avant l'éruption et pendant celle-ci, de façon directement proportionnelle à l'augmentation des ostéoclastes dans les zones adjacentes à la crypte contenant le follicule dentaire. L'afflux des cellules mononuclées diminue ensuite, parallèlement au nombre des ostéoclastes.

En 1985, des études en microscopie électronique confirment la présence des cellules mononuclées et précisent qu'elles ressemblent à des monocytes qui contiennent des granules caractéristiques des pré-ostéoclastes [10]. Ces expériences furent interprétées comme suit :

- le follicule, qui possède une microvascularisation, attire en son sein des monocytes ;

- ceux-ci migrent ensuite vers les parois de la crypte contenant le follicule dentaire et se différencient en ostéoclastes pour participer à la réalisation du chemin d'éruption.

En utilisant la microscopie électronique et des marqueurs spécifiques des ostéoclastes, l'équipe de Marks a pu montrer que le déplacement de la crypte osseuse était bien dû à un phénomène ostéoclasique et non à une simple croissance des maxillaires ou à une pression exercée par la dent [11]. Ils ont même pu démontrer que le processus ostéolyse-ostéoformation est polarisé (ostéoclasie coronaire et ostéogenèse apicale), et qu'il subit une régulation bilatérale, symétrique, et locale de la part du follicule [12].

Entre temps, Marks et Cahill vont mener une expérience originale, qui marque un tournant dans la recherche sur l'éruption et qui va balayer tous les anciens concepts [13]. Ils substituent à la dent, avant le début de l'éruption, un objet inerte : les couronnes furent donc extraites et remplacées dans le follicule dentaire par des couronnes dévitalisées ou par des répliques en métal ou en silicone. Les études radiologiques et histologiques ont alors montré que ces ersatz de dent faisaient leur éruption normalement, médecine buccale chirurgie buccale VOL. $9, \mathrm{~N}^{\circ}$ 2003 page 97 
médecine buccale chirurgie buccale après création du chemin d'éruption et formation de trabécules osseux à la base de la crypte. L'évolution est identique si les couronnes ne sont pas remplacées. Ils en concluent que le follicule est nécessaire à l'éruption ; cette demière résulte d'un processus conjuguant ostéolyse et ostéoformation aux extrémités opposées du follicule, et que la dent elle-même ne joue aucun rôle dans le processus.

Afin de préciser le rôle du follicule, ils réalisent en 1987 l'ablation sélective de ses différentes parties. La résection de la partie coronaire entraîne l'absence d'ostéolyse et donc du chemin d'éruption; la résection de la partie basale l'absence d'ostéoformation. Dans les deux cas, l'éruption n'a pas lieu. Cette expérience montre que l'ostéolyse et l'ostéoformation sont polarisées et régulées localement par la partie adjacente du follicule dentaire [12].

Au début des années 90, l'équipe de Marks a acquis suffisamment de données expérimentales pour prouver le rôle de l'ostéolyse dans l'éruption [14]. Ce rôle est confirmé par Sundquist et Marks en utilisant de la bafilomycine $A 1$, un inhibiteur spécifique de la pompe à protons membranaires des ostéoclastes. En bloquant l'ostéolyse, elle empêche l'éruption de façon très localisée [15]. En 1998, Grier et Wise obtiennent le même résultat avec un biphosphonate, substance qui inhibe l'activité ostéoclastique des ostéoclastes [16]. En 1999, l'étude des caractéristiques phénotypiques des cellules du follicule dentaire permet de montrer qu'il y a une relation génétique entre les cellules du follicule dentaire et la formation de tissu minéralisé péri-folliculaire. Les marqueurs cyto chimiques exprimés par ces cellules mises en culture correspondent à ceux des tissus minéralisés (ostéopontine, collagène de type I et III, sialoprotéine osseuse, phosphatase alcaline, fibronectine) [17].

\section{LES FACTEURS DE CROISSANCE}

Ils interviennent de façon prépondérante dans la morphogenèse crânio-faciale et dentaire [18]. La compréhension de la régulation de l'expression de leurs gènes constitue sans doute une étape importante pour mieux comprendre les mécanismes qui interviennent dans l'éruption dentaire. Wise et coll., en 1992, mettent au point une méthode pour la culture des cellules du follicule dentaire afin de tester la réponse des cellules du follicule aux facteurs de croissance intervenant dans l'éruption [19]. Le phénomène ostéoclasique, indispensable à la réalisation du chemin d'éruption, nécessite un afflux de monocytes dans le follicule et certains se transforment en ostéoclastes. Quels sont les signaux moléculaires à l'origine de ces évènements ?

\section{Epidermal Growth Factor (EGF)}

L'EGF a des propriétés mitogéniques et intervient dans la différentiation de nombreux tissus et organes [20]. Wise et coll., en 1992, étudient grâce à des techniques immunohistochimiques, la localisation de l'EGF et de ses récepteurs chez le rat avant et pendant l'éruption [21]. II existe une corrélation entre les évènements moléculaires et cellulaires intervenant dans l'éruption. L'action de l'EGF se produit pendant I'afflux des monocytes. L'EGF pourrait provenir d'une source endogène (le follicule et l'organe de l'émail), l'éruption serait alors régulée de façon auto- ou paracrine [21]. Lin [22] et Shroff [23] montrent que les récepteurs de l'EGF sont également transcrits et traduits dans le for licule dentaire, ce qui a pour effet d'entraîner une accélération de l'éruption. L'EGF a donc de nombreux rôles dans l'éruption, ce qui est confirmé par Klein, qui montre l'action de l'EGF sur les hormones stéroïdiennes impliquées dans la croissance comme dans l'éruption dentaire [24]. La stimulation de l'éruption, notamment celle induite par l'EGF, résulte de plusieurs facteurs : en dehors de la prolifération cellulaire et de l'afflux de monocytes dans le follicule, il y a la synthèse du collagène, le remodelage tissulaire et vasculaire... II devient évident que I'EGF agit à différents niveaux du processus complexe de l'éruption et qu'il interagit avec des cytokines et d'autres facteurs de croissance.

Transforming Growth Factor - $ß$ (TGF-ß) L'afflux de monocytes dans le follicule dentaire a lieu au $3^{e}$ jour pour les molaires de rat, or 
TGF-ß a un effet chimiotactique sur les monocytes in vitro. Les études immunocytochimiques ont montré qu'il est présent dans le réticulum étoilé pendant les deux premiers jours, puis il disparaît. II est absent dans les autres structures participant au processus d'éruption, y compris dans le follicule dentaire. La présence de TGF-ß juste avant I'afflux monocytaire fait suspecter que le TGF-ß pourrait favoriser l'afflux de monocytes du sang périphérique vers le follicule [25].

\section{Colony Stimulating Factor - One (CSF-1)}

Le Macrophage Colony Stimulating Factor (MCSF) ou CSF-1 qui est absent chez la souris ostéopétrotique, est nécessaire à la différentiation et à la maturation des monocytes [26]. En 1992, lizuka réalise une expérience sur des rats ostéopétrotiques sans dents (toothless rats $=$ [t l] ) [6]. Ils sont issus d'une mutation ostéopétrotique caractérisée par une sclérose squelettique généralisée, une ostéoclasie très réduite, avec peu d'ostéoclastes et une absence totale d'éruption des dents. Contrairement aux autres formes d'ostéopétrose, celle-ci n'est pas curable par une greffe de moelle osseuse. Cependant, les anomalies squelettiques sont considérablement améliorés par un traitement à base de CSF-1. L'absence totale d'éruption chez les rats [ $\mathrm{t} l]$ est donc directement lié à l'ostéoclasie réduite, caractéristique de cette anomalie. L'injection néonatale de CSF-1, en permettant la lyse osseuse, entraîne une éruption dentaire normale. L'injection de CSF-1 doit être faite à la naissance : si elle n'est pas faite précocement il y a ankylose et absence d'éruption des dents. D'autre part, les enzymes liés à la fonction ostéoclasique sont élevés chez les rats [ $\mathrm{t}$ l] traités ce qui laisse penser que CSF-1 favorise la transformation des monocytes en ostéoclastes [6,27].

Les préostéoclastes, précurseurs des ostéoclastes, apparaissent précocement dans le follicule dentaire et précèdent l'augmentation des ostéoclastes dans l'os alvéolaire adjacent. A partir de 1994, de nombreuses études examinent les effets de l'administration de CSF-1 sur l'éruption des premières molaires de rats $[t]$, et sur les ostéoclastes et leurs précurseurs proches d'une dent en éruption [28,8]. Ces études montrent que le CSF-1 accélère l'éruption chez les rats témoins en augmentant le nombre des ostéoclastes et de leurs précurseurs [29]. Ceci suggère que l'ostéoclasie est un processus important dans la phase précoce de l'éruption et son augmentation accélère l'éruption. Le CSF-1 joue donc un rôle dans le métabolisme moléculaire régulant l'éruption et son administration locale pourrait être utile pour favoriser l'éruption et le déplacement des dents [28].

Wise et Lin démontrent que les cellules du follicule transcrivent et traduisent I'ARNm du CSF-1 et que le CSF-1 a un effet autocrine sur la transcription de ses propres gènes [30]. Par des techniques d'immunofluorescence, une autre étude va révéler que l'expression maximale du CSF-1 et l'afflux maximal des monocytes sont concomitants, et que le CSF-1 contribuerait à cet afflux [31].

Ces études établissent donc une relation entre le CSF-1, les monocytes et le follicule qui semble tenir un rôle primordial dans l'éruption. Si le CSF-1 représente le signal initiant l'éruption, il reste à préciser le lieu et le mode de régulation de sa synthèse.

\section{Interleukine-1 $\alpha$ (IL-1 $\alpha$ )}

Elle stimule la transcription du gène du CSF-1 sur un mode temps et concentration dépendant [30]. La régulation de l'expression du gène du CSF-1 par IL- $1 \alpha$ ainsi que par le CSF-1 luimême, intervient dans le signal du début de l'éruption. L'action de I'IL-1 $\alpha$ sur le CSF-1 est clairement démontrée in vivo en 1998 par Wise [32]. L'importance de l'IL- $1 \alpha$ a d'ailleurs été récemment confirmée par l'éruption retardée observée chez des souris n'exprimant pas le récepteur de I' IL-1 $\alpha$; les gènes du CSFI sont toujours exprimés mais c'est une autre molécule qui initie leur expression. L'IL-1 $\alpha$ joue bien un rôle dans l'éruption puisque, en son absence, l'éruption est retardée ; cependant, elle peut avoir lieu sans le signal donné par I'IL-1 $\alpha$ [33]. médecine buccale chirurgie buccale VOL. $9, \mathrm{~N}^{\circ}$ 2003 page 99 
médecine buccale chirurgie buccale
Sachant que I'IL- $1 \alpha$ stimule la transcription du gène du CSF-1 in vitro, $y$-a-t-il des facteurs régulant I'IL-1 $\alpha$ ? Les techniques d'immunofluorescence ont permis de localiser l'IL- $1 \alpha$ dans le réticulum étoilé. L'injection d'EGF augmente la quantité d' IL- $1 \alpha$ dans le réticulum étoilé : I'EGF stimule la traduction du gène de l'IL- $1 \alpha$ dans le réticulum étoilé et pourrait donc initier une cascade de signaux moléculaires marquant le début de l'éruption dentaire [34,35]. Cette hypothèse est confirmée par Wise en 1997 qui précise que le TGF-ß a la même action que l'EGF car il utilise les mêmes récepteurs [36, 37].

L'EGF et le CSF-1 n'ont d'ailleurs pas le même impact sur l'éruption selon qu'il s'agisse des incisives ou des molaires du rat. En effet, l'EGF accélère l'éruption des incisives mais n'a pas d'effet très significatif sur celle des molaires ; au contraire, le CSF-1 accélère celle des molaires plutôt que celle des incisives. La dexaméthasone, connue pour augmenter la résorption osseuse in vivo et in vitro, donne les mêmes résultats que l'EGF [38]. Les incisives et les molaires des rongeurs pourraient donc répondre préférentiellement aux différentes molécules de régulation [39]. Comme l'éruption des incisives du rat est continue et celle des molaires limitée, il paraît difficile d'extrapoler ces résultats à la dentition humaine.

\section{Monocyte Chemotactic Protein One (MCP-1)}

Il a été démontré que l'éruption dentaire nécessitait un afflux de monocytes dans le follicule dentaire qui, sous la forme d'ostéoclastes, aménageront le chemin d'éruption [10]. Comme le CSF-1, la MCP-1 attire les monocytes par chimiotactisme mais son rôle dans l'éruption reste encore imprécis. Comment cette molécule intervient-elle auprès des monocytes ? II semble que le CSF-1 stimule l'expression de la MCP-1 dans les cellules endothéliales et l'adhérence des monocytes aux cellules endothéliales serait favorisée par le CSF-1 et inhibée par un anticorps spécifique de la MCP-1 [40,41].

Que et Wise en 1997 et 1998, par la technique de transcription inverse, ont montré que les cellules du follicule dentaire transcrivent le gène de
MCP-1 et que le pic d'expression correspond à l'afflux monocytaire. Ils ont montré aussi que toutes les molécules favorisant l'éruption ( $E G F$, IL- $1 \alpha, T G F-\beta$, et CSF-1) augmentent l'expression du gène de MCP-1 dans le follicule [42,43]. Ainsi, in vivo, I'IL- $1 \alpha$ et l'EGF qui augmentent I'expression de MCP-1 apparaissent avoir une action redondante.

\section{C-fos}

Le c-fos, retrouvé dans le follicule, est également susceptible d'intervenir dans le processus d'éruption. II s'agit d'une protéine impliquée dans le processus mitogénique des facteurs de croissance et nécessaire à la différenciation de certaines cellules comme les ostéoclastes.

Le follicule constituerait une source endogène de c-fos, ce qui apparaît normal puisque cette protéine est présente dans les cultures de fibroblastes dérivant d'autres organes. Ceci suggère que c-fos joue un rôle dans l'éruption, probablement en régulant la différentiation des monocytes en ostéoclastes. Cette hypothèse est basée sur les faits suivants :

- la présence de c-fos dans les tissus nécessaires à l'éruption et au moment de l'afflux des cellules monocytaires dans le follicule ;

- le c-fos est nécessaire à la production des ostéoclastes [44];

- I'EGF et le CSF-1 qui accélèrent l'éruption, augmentent l'expression du gène $c$-fos $[45,46]$; - les souris mutantes pour c-fos, et qui n'expriment pas cette protéine, n'ont pas d'ostéoclastes ni d'éruption dentaire ; la différenciation des ostéoclastes peut être rétablie par transplantation de moelle osseuse normale.

Enfin, des expériences de "clivage génique » ont montré que la rupture de c-fos contribue à un phénotype d'ostéopétrose caractérisé par une absence complète d'ostéoclastes.

Le défaut est intrinsèque aux précurseurs hématopoïétiques, incapables d'évoluer à un stade précoce de différentiation en ostéoclastes [8].

\section{Parathormone (PTH) et Parathyroide Hormone related Protein (PTHrP)}

L'os alvéolaire est soumis aux mêmes facteurs de régulation systémique pour l'ostéoclasie que le reste du squelette. La PTH représente le prin- 
cipal inducteur d'ostéoclasie en stimulant la formation des ostéoclastes.

L'application locale et prolongée de PTH, sous forme d'un gel de PTH-méthylcellulose, entraîne une accélération dose-dépendante des mouvements orthodontiques. Les traitements orthodontiques pourraient bénéficier de cette propriété dans les prochaines années [47].

Un peptide apparenté à la PTH peut se lier au récepteur de la PTH et mimer ses effets osseux.

Des études récentes indiquent que ce peptide, la PTHrP, est nécessaire à l'éruption chez la souris [48], Que et Wise ont montré que PTHrP améliore I'expression du CSF-1 et de la MCP-1, toujours sur un mode temps et concentration dépendante. De plus, I'IL1- $\alpha$ augmente I'expression des gènes du récepteur de PTHrP dans les cellules du follicule dentaire [49]. Et Nakchbandi a montré que la PTHrP est nécessaire à la formation et à la différenciation des ostéoclastes, et qu'elle diminue l'expression des facteurs inhibant l'ostéoclastogenèse dans le follicule dentaire [50].

\section{Nuclear Factor - kappa B (NF-kB)}

II s'agit d'un facteur de transcription nucléaire qui pourrait participer à la séquence des signaux moléculaires de l'éruption. Les travaux de Que et coll. montrent que le NF-kB est exprimé et synthétisé par les cellules de follicule dentaire en culture. De plus, sa transcription, son activation et sa translocation sont améliorées par l'IL- $1 \alpha$. In vivo, le NF-kB est exprimé de façon maximale dans le follicule au moment de l'afflux de monocytes dans le follicule. Quoiqu'il en soit, le rôle exact de NF-kB dans l'éruption n'est pas entièrement élucidé mais il pourrait constituer un des facteurs clef de l'éruption dentaire [51].

\section{CONCLUSION}

Les recherches s'orientent vers une théorie multifactorielle de l'éruption dentaire, dans laquelle de nombreuses molécules entrent en jeu, à des stades différents, parfois de façon redondante.

Certaines molécules vont stimuler l'influx monocytaire nécessaire à la formation des ostéoclastes, tels le CSF-1, la MCP-1, le c-fos et le NF-kB [52]. D'autres vont à la fois, plus ou moins directement, faciliter l'expression des molécules précédentes et stimuler les ostéoclastes : I'EGF, I'IL- $1 \alpha$, le TGF- $\beta$ et la PTHrP.

Cependant, les théories de l'éruption dentaire comportent toutes une notion identique : I'ostéolyse est indispensable à l'éruption dentaire. Cette idée a mené les auteurs à formuler, dès les années 90 , une théorie s'intéressant à la biologie moléculaire locale [53,54]. II fallait donc déterminer les facteurs d'éruption ayant un rôle physiologique, afin de tester leur action sur les dents incluses ou ayant un retard d'éruption, ainsi que sur le turn-over de l'os alvéolaire. Ces recherches ouvrent aussi de nouvelles perspectives pour les traitements orthodontiques, dont la réalisation pourrait être facilitée. Cependant les applications thérapeutiques sont actuellement limitées car la plupart des recherches n'ont encore pas dépassé le stade de l'expérimentation animale.

\section{RÉFÉRENCES}

1 - CAHILL DR. Eruption pathway formation in the presence of experimental tooth impaction in puppies. Anat Rec 1969; 164: 67-78.

2 - CAhill DR, Marks SC. Chronology and histology of exfoliation and eruption of mandibular premolars in dogs. J Morph 1982; 17: 213-8.

3 - CahILL DR, Marks SC. Tooth eruption: evidence of the central role of the dental follicle. J Oral Pathol 1980; 9: 189-200.

4 - Larson EK, Cahill DR, Gorski JP, Marks SC. The effect of removing the true dental follicle on premolar eruption in the dog. Arch Oral Biol 1994; 39: 271-5.

5 - MARKS SC. Tooth eruption depends on bone resorption: experimental evidence from osteopetrotic [ia] rats. Metab bone Dis Relat Res 1981; 3: 107-15.

6 - Iizuka T, Cielinski M, Aukerman SL, Marks SC. The effects of CSF-1 on tooth eruption in the toothless (osteopetrotic) rat in relation to the critical periods for

médecine buccale chirurgie buccale VOL. $9, \mathrm{~N}^{\circ}$ 2003 page 101 
bone resorption during tooth eruption. Arch Oral Biol 1992; 37: 629-36.

7 - Cielinski MJ , IIZUKA T, M ARKS SC. Dental abnormality in the osteopetrotic rat mutation microphtalmia blanc. Arch Oral Biol 1994; 39: 985-90

8 - Felix R, Hofstetter W, CeChini MG. Recent developments in understanding of pathophysiology of osteopetrosis. Eur J Endocrinol 1996; 134: 143-56.

9 - Marks SC, Cahill DR, Wise GE. The cytology of the dental follicle and adjacent alveolar bone during tooth eruption in the dog. Am J Anat 1983; 168: 277-89.

10 - Wise GE, Marks SC, CahiLl DR. Ultrastructural feature of the dental follicle associated with formation of the tooth eruption pathway in the dog. J Oral Pathol Med 1985; 14: 15-26.

11 - MARKS SC, CAHILL DR. Ultrastructure of alveolar bone during tooth eruption in dog. Am J Anat 1986; 177: 427-38.

12 - Marks SC, CahILL DR. Regional control by the dental follicle of alterations in alveolar bone metabolism during tooth eruption. J Oral Pathol 1987; 16: 164-9.

médecine

buccale

chirurgie

buccale

L. $9, N^{\circ} 2$

2003

page 102

13 - MARKS SC, C AHILL DR. Experimental study in the dog of the non-active rôle of the tooth in the eruptive process. Arch Oral Biol 1984; 29: 311-22.

14 - Marks SC, Cielinski MJ, Sundquist K, Wise GE, GORSKI J P. The role of bone resorption in tooth eruption (pp 483-8). In: The biological mechanisms of tooth eruption, resorption and replacement by implants. Davidovitch Z ed., AL: EBSCO Media, Birmingham, 1994.

15 - SUNDQUIST KT, MaRKS SC. Bafilomycin A1 inhibits bone resorption and tooth eruption in vivo. J Bone Miner Res 1994; 10: 575-82.

16 - GRIER RL, WISE GE. Inhibition of tooth eruption in the rat by a biphosphonate. J Dent Res 1998; 77: 8-15.

17 - HOU LT, LIU CM, Chen YJ, WONg MY, Chen KC, CHEN J, THOMAS HF. Characterization of dental follicle cells in developing mouse molar. Arch Oral Biol 1999; 44: 759-70.

18 - TheslefF I. Homeobox genes and growth factor in regulation of craniofacial and tooth morphogenesis. Acta Odontol Scand 1995; 53: 129-34.

19 - WISE GE, LIN F, FAN W. Culture and characterization of dental follicle cells from rat molars. Cell Tissue Res 1992; 267: 43-92.

20 - COHEn S. The stimulation of epidermal proliferation by a specific protein (EGF). Dev Biol 1965; 12: 394-407.

21 - WISE GE, LIN F, FAN W. Localization of epidermal growth factor and its receptor in mandibular molars of the rat prior to and during prefunctional tooth eruption. Dev Dyn 1992; 195: 121-6.

22 - LIN F, ZHAO L, WISE GE. In vivo and in vitro effects of EGF on its gene expression in rat dental follicle cells. Arch Oral Biol 1996; 41: 485-91.

23 - Shroff B, Kashner JE, Keyser J D, Hebert C, NoRRIS K. EGF and EGF receptor expression in mouse dental follicle during tooth eruption. Arch Oral Biol 1996; 41: 613-7.

24 - Klein RM, Chiego DJ, Sonneborn AA, Tophan RT, GatTONE VH. Effects of growth factors on tooth eruption and related developmental process (pp 407-28). In: The biological mechanisms of tooth eruption, resorption and replacement by implants. Davidovitch $Z$ ed., AL : EBSCO Media, Birmingham, 1994.

25 - WISE GE, FAN W. Immunolocalization of transforming growth factor $ß$ in rat molars. J Oral Pathol Med 1991; 20: 74-80.

26 - Stanley ER, Guilbert LJ , TUShinski RJ , Bartelmez SH. CSF-1: a mononuclear phagocyte lineage-specific hematopoietic growth factor. J Cell Biochem 1983; 21: 151-9.

27 - Nidda S, Abe M, Suemune S, Yoshiko Y, Maeda N, YAMASAKI A. Restoration of disturbed tooth eruption in osteopetrotic (op/op) mice by injection of MCSF. Exp Anim 1997; 46: 95-101.

28 - Cielinski NJ , J olie M, Wise GE, Ando DG, Marks SC. Colony stimulating factor- 1 is a potent stimulator of tooth eruption in the rat. In: The biological mechanisms of tooth eruption, resorption and replacement by implants. Davidovitch Z ed., AL : EBSCO Media, Birmingham, 1994, pp 429-36.

29 - Marks SC, W oj TOWicz A, S Zperl M, U Rbanowska E, Mackay CA， WikTOR-J EDRZEjCZAK W, Stanley ER, AUKERMAN SL. Administration of CSF-1 corrects some macrophage, dental and skeletal defects in an osteopetrotic mutation (toothless, $\mathrm{t}$ ) in the rat. Bone 1992; 13: 89-93.

30 - WISE GE, LIN F. Regulation and localization of CSF-1 m-RNA in cultured rat dental follicle cells. Arch Oral Biol 1994; 39: 621-7.

31 - WISE GE, LIN F, ZHAO L. Transcription and translation of CSF-1 in the dental follicle. J Dent Res 1995; 74: 1531-7.

32 - WISE GE. In vivo effects of IL1- $\alpha$ on CSF-1 gene expression in the dental follicle of rat molar. Arch Oral Biol 1998; 43: 163-5.

33 - HUANG H, WISE GE. Delay of tooth eruption in null mice devoid of the type I IL-1R gene. Eur J Oral Sci 2000; 108: 297-302.

34 - MARKS SC. The basic and applied biology of tooth eruption. Connect Tissue Res 1995; 32: 149-57.

35 - WISE GE, LIN F, ZHAO L. Immunolocalization of IL1 $\alpha$ in rat mandibular molars and its enhancement after in vivo injection of EGF. Cell Tissue Res 1995; 280: 21-6.

36 - WISE GE, ZHAO L. Immunostaining and transcription enhancement of $\mathrm{IL}-1$ receptor type- 1 in the rat dental follicle. Arch Oral Biol 1997; 42: 339-44.

37 - HUANG H, WISE GE. Delay of tooth eruption in null mice devoid of the type I IL-1 gene. Eur J Oral Sci 2000; 108: 297-302.

38 - WiSE GE, GRier RL, LMpkin SJ , Zhang Q. Effects of dexamethasone on tooth eruption in rats: differences 
in incisor and molar eruption. Clin Anat 2001; 14: 204-9.

39 - Cielinski MJ, J olie M, Wise Ge, Marks SC. The contrasting effects of CSF-1 and EGF on tooth eruption in the rat. Connect T Res 1995; 32: 165-9.

40 - Cielinski MJ , J olie M, Marks SC. The role of CSF-1 in osteoclast recruitment during tooth eruption. J Dent Res 1993; 72: 283.

41 - Shyy YJ, Wickham LL, Hagan JP, Hsieh HJ , Hu YL, Telian SH, Valente AJ, Sung KLP, Chien S. Human MCSF stimulates the gene expression of MCP-1 and increases the adhesion of monocytes to endothelial monolayers. J Clin Invest 1993; 92: 1745-51.

42 - QUe BG, WISE GE. Colony stimulating factor 1 and monocyte chemotactic protein-1 chemotaxis for monocytes in the rat dental follicle. Arch Oral Biol 1997; 42: 855-86.

43 - QUE BG, WISE GE. Tooth eruption molecules enhance MCP-1 gene expression in the dental follicle of the rat. Dev Dyn 1998; 212: 346-51.

44 - Grigoriadis AE, Wang ZQ, Cecchini MG, Hofstetter W, Felix R, Fleisch HA, Wagner EF. Cfos: a key regulator of osteoclast-macrophage lineage determination and bone remodeling. Science 1994; 266: 443-8.

45 - WISE GE. The biology of tooth eruption. J Dent Res 1998; 77: 1576-9.

46 - WISE GE, LIN F, ZHAO L. Effects of EGF and CSF-1 on expression of $\mathrm{C}$-fos in rat mandibular molars : implication for tooth eruption. Cell Tissue Res 1996; 284: 1-7.
47 - Soma S, Matsumoto S, Higuchi Y, TaKano-Yamamoto T, YAMASHITA K, KURISU K, IWAMOTO M. Local and chronic application of PTH accelerates tooth movements in rats. J Dent Res 2000; 79: 1717-24.

48 - Philbrick WM, Dreyer BE, Nakchbandi IA, Karapli AC. Proc Natl Acad Sci 1998; 95: 11846-51.

49- Wise GE, Que BG, Huang H, Lumpkin SJ . Enhancement of gene expression in rat dental follicle cells by parathyroid hormone-related protein. Arch Oral Biol 2000; 45: 903-9.

50 - Nakchbandi IA, Weir EE, Insogna KL, P hilbrick WM, BRoAdus AE. Parathyroid hormone-related protein induces spontaneous osteoclast formation via a paracrine cascade. Proc Natl Acad Sci 2000; 97: 7296-300.

51 - Que BG, LUMPkin, WISE GE. Implication for tooth eruption of the effect of IL-1 $\alpha$ on Nuclear-Factor-KB gene expression in the rat dental follicle. Arch Oral Biol 1999; 44: 961.

52- Wise GE, HuAng H, Que BG. Gene expression of potential tooth eruption molecules in the dental follicle of the mouse. Eur J Oral Sci 1999; 107: 482-6.

53 - WISE GE, LIN F, MARKS SC, GORSKI J P. The molecular basis of tooth eruption. In: The biological mechanisms of tooth eruption, resorption and replacement by implants. Davidovitch Z ed., AL : EBSCO Media, Birmingham, 1994, pp 383-9.

54 - Marks SC, Schroeder HE. Tooth eruption: theories and facts. Anat Rec 1996; 245: 374-93. médecine buccale chirurgie buccale VOL. $9, \mathrm{~N}^{\circ}$ 2003 page 103 